\section{A case report of an Erdheim-Chester patient focused on pain management}

\author{
José Osvaldo Barbosa Neto1*, Joao Batista Santos Garcia1, \\ Thiago Alves Rodrigues ${ }^{1}$ and Camila Freitas de Andrade \\ Rodrigues $^{2}$
}

${ }^{1}$ Experimental Laboratory to the Study of Pain, Federal University of Maranhão, Brazil ${ }^{2}$ Federal University of Maranhão, Brazil

\section{Summary}

Introduction: Erdheim-Chester disease (ECD) is a rare and difficult-to-treat non-Langerhans cell histiocytosis characterized by the excessive production and accumulation of histiocytes. This study reports a case of ECD, emphasizing both its diagnosis, assessment and treatment of the pain associated with the disease.

Case Report: Six years ago, a 39-year-old male patient presented with generalized pain of moderate intensity in the lower limbs that involved periods of greater intensity associated with ambulation. The diagnosis of histiocytosis associated with panhypopituitarism and adrenal insufficiency was proposed. For a specific diagnosis, a bone lesion biopsy was performed, revealing the presence of histiocytic proliferation that was CD1 negative, S100 protein positive, and CD68 negative. Therefore, the diagnosis of non-Langerhans histiocytosis known as ECD was confirmed. During the two years that followed, the patient presented with severe bone pain, particularly in the lower limbs and cranial vault, and the pain subsided to a certain extent with the use of tramadol and paracetamol. Because of the pain, the patient was unable to walk and became bedridden As the patient remained in severe pain, even after the administration of morphine, the opioid was changed from morphine $(60 \mathrm{mg} /$ day) to oxycodone $(30 \mathrm{mg} /$ day) for a convenient dosing schedule; furthermore, the oxycodone dosage was scheduled to increase to $40 \mathrm{mg} /$ day that same week. The patient experienced significant pain reduction, requiring rescue analgesia only once or twice a week.

Conclusion: To the best of our knowledge, this is the first case report on the characterization and treatment of pain specific to ECD, and we highlight that the patient had a good response to treatment.

\section{More Information}

*Address for Correspondence: José Osvaldo Barbosa Neto, Experimental Laboratory to the Study of Pain-Federal University of Maranhão, Brazil, Tel: +5598991675217; Email: osvaldo1983@me.com; osvbarbosa@yahoo.com.br

Submitted: 12 July 2019

Approved: 11 September 2019

Published: 12 September 2019

How to cite this article: Neto JOB, Garcia JBS, Rodrigues TA, de Andrade Rodrigues CF. A case report of an Erdheim-Chester patient focused on pain management. J Hematol Clin Res. 2019; 3: 060-063.

DOI: dx.doi.org/10.29328/journal.jhcr.1001013

Copyright: @ 2019 Neto JOB, et al. This is an open access article distributed under the Creative Commons Attribution License, which permits unrestricted use, distribution, and reproduction in any medium, provided the original work is properly cited

Keywords: Pain management; Cancer pain Erdheim-Chester Disease; Histiocytosis, NonLangerhans-Cell

Check for updates

\section{Introduction}

Erdheim-Chester disease (ECD) is a rare non-Langerhans cell histiocytosis characterized by the excessive production and accumulation of histiocytes [1]. The cause of the disease remains unknown; for this reason, ECD is not classified as an autoimmune disease, storage disorder, infection, or cancer. However, the rapid proliferation of histiocytes, with infiltration of the bone marrow and rapid dissemination throughout the body, allows the classification of ECD as an aggressive form of cancer [1-3]. ECD is most common in middle-aged men and involves the impairment of the loose connective tissues, endocrine glands, skin, pituitary glands, kidneys, pericardium, lungs, and brain. The disease initially affects the skeletal system, leading to an abnormal increase in bone density and fibrosis, followed by severe pain. In most cases, bone pain is the first symptom of the disease. In addition, the control of ECD can be difficult and expensive [2-5]. Both the rarity of ECD and the range of affected organs contribute to the difficulty of the diagnosis, which is performed by tissue biopsy and reveals histiocytic infiltration. ECD is considered incurable, and its treatment is not currently well established [6]. Unfortunately, about $60 \%$ of patients succumb to their disease within 32 months of presentation [7]. Of interest, researchers reported that interferon- $\alpha$ results in terminal differentiation of histiocytes and dendritic cells [8,9]. This study reports a case of ECD, emphasizing both its diagnosis, assessment and treatment of the pain associated with the disease.

\section{Case Report}

Six years ago, a 39-year-old male patient presented with 
generalized pain of moderate intensity in the lower limbs that involved periods of greater intensity associated with ambulation. Moreover, the patient presented with episodes of moderate unilateral non-throbbing headache in the left hemicranium. However, only after approximately three years, the patient sought medical assistance for the disease diagnosis. The clinical examination indicated the presence of xanthelasmas, bilateral exophthalmos, the absence of focal neurological signs, and the absence of pain during the clinical examination. During the monitoring period, imaging exams were performed. The computed tomography (CT) revealed scleroticlesions in the left iliac, right sacrum, vertebral bodies, and diffusely in the ribs. The scintigraphy of the skeleton indicated radiotracer uptake in the distal thirds of the tibia, the bilateral maxilla, and the cranial vault. With regard to the radiological investigation, positron emission tomography (PET) scan showed a marked uptake of fludeoxyglucose (FDG) in the distal thirds of both femurs, proximal third of the left femur, proximal fibula, foot, and ankle. Nuclear magnetic resonance imaging (MRI) of the brain indicated nodular thickening of the pituitary stalk and bilateral proptosis (grade I) in the eyes. The diagnosis of histiocytosis associated with panhypopituitarism and adrenal insufficiency was proposed. For a specific diagnosis, a bone lesion biopsy was performed, revealing the presence of histiocytic proliferation that was CD1 negative, S100 protein positive, and CD68 negative. Therefore, the diagnosis of non-Langerhans histiocytosis known as ECD was confirmed. The treatment was initiated immediately, with three million IU of interferon- $\alpha$ three times per week and hormonal supplementation monitored by an endocrinologist. However, the patient was treated for only three months, after which he abandoned the treatment.

During the two years that followed, the patient presented with severe bone pain, particularly in the lower limbs and cranial vault, and the pain subsided to a certain extent with the use of tramadol and paracetamol. Because of the pain, the patient was unable to walk and became bedridden. After this two-year period without treatment, the patient was submitted to imaging examination of the pituitary and the axial and appendicular skeleton by PET scan, which revealed an increase in glycolytic metabolism, corroborating the diagnosis of ECD's activity. The interferon therapy was promptly resumed in addition to $10 \mathrm{mg}$ morphine administered every $4 \mathrm{~h}$, and the patient was referred to a pain management program. During the specialized evaluation, the patient complained of intense pain for approximately 1.5 years. Using the Numerical Rating Scale (NRS) at the time of consultation, the pain was rated with a score of 8 in the lower limbs, back, and skull. This pain was continuous, throbbing, and severe, with periods of exacerbation, and would improve with rest and with the use of morphine. The patient also presented with depressed mood and anxiety, having thoughts about death, and complained of constant asthenia that worsened during the administration of interferon- $\alpha$. Furthermore, he reported a lack of enthusiasm to perform routine activities or work. On physical examination, he exhibited no physical limitations in the limbs, focal neurological signs, pain during clinical examination, signs of immobility, or dystrophies.

At this time, the patient was diagnosed with nociceptive cancer pain associated with bone injury in multiple locations and exhibited depressive-anxious symptomatology. As the patient remained in severe pain, even after the administration of morphine, the opioid was changed from morphine $60 \mathrm{mg} /$ day) to oxycodone $(30 \mathrm{mg} /$ day) for a convenient dosing schedule; furthermore, the oxycodone dosage was scheduled to increase to $40 \mathrm{mg} /$ day that same week. The following adjuvants were administered: paracetamol $(500 \mathrm{mg})$ every $6 \mathrm{~h}$, ketoprofen $(100 \mathrm{mg}$ ) every $12 \mathrm{~h}$ (maintained at this dosage for 10 days), rescue analgesia with morphine $(10 \mathrm{mg})$ administered orally, and sertraline $(50 \mathrm{mg} /$ day) for the treatment of depression. In addition, laxatives and anti-emetic agents were included as part of the symptom management. The patient was referred for physical rehabilitation and nutritional and psychiatric evaluation. After consulting with a psychiatrist, the sertraline dosage was maintained, and the use of venlafaxine $(150 \mathrm{mg} /$ day $)$ was initiated for the treatment of depression. The patient experienced significant pain reduction, requiring rescue analgesia only once or twice a week. This ECD case was reevaluated monthly without modifications for eight consecutive months. In the ninth month of treatment in the pain management program, the patient developed refractory constipation with the use of the laxatives bisacodyl and lactulose, requiring the frequent use of glycerin enemas. Thus, the pain treatment was changed to transdermal fentanyl $(25 \mu \mathrm{g} / \mathrm{h})$ while maintaining the adjuvants. After the latter treatment, the patient experienced less constipation, and the pain was under control, requiring a morphine rescue only once or twice a week. He continues treatment with monitoring by a multidisciplinary team.

\section{Discussion}

This study reports one case of Erdheim-Chester disease, with a view to the diagnosis and treatment of pain. ECD is a rare systemic disorder of non-Langerhans histiocytic cells and was first described by Jacob Erdheim and William Chester in 1930 [10,11]. Between 1930 and January 2013, a little more than 500 cases have been described [12]. ECD affects primarily middle-aged men and has heterogeneous clinical manifestations, ranging from asymptomatic cases and bone injuries to multiple organ involvement; with a five-year survival rate of $68 \%$ [13]. Virtually any tissue can be affected. However, bones, the central nervous system, and the retroperitoneum are most often affected [14]. This case report presents a 39-year-old male patient presenting with infiltrations in the three regions most often affected - long bones, the pituitary stalk, and the adrenal glands. Consequently, the spectrum of clinical manifestations in this patient involved not only the bone structures but also 
various endocrine changes because of the compromised synthesis of pituitary and adrenal hormones. Considering the heterogeneous clinical manifestations, the diagnosis of ECD requires a high index of suspicion and must be ascertained by additional examinations. In this sense, imaging examinations have greatly contributed to confirming the diagnosis [13]. In the radiography of long bones, bilateral cortical sclerosis involving diametaphyseal regions can be identified and is considered pathognomonic [1]. In addition to radiography, unusually intense Tc- $99 \mathrm{~m}$ activity at the distal ends of long bones detected by bone scintigraphy is also indicative of ECD [1]. Lastly, the diagnosis should be based on typical histological findings, indicating the infiltration of foamy histiocytes nested along polymorphic granuloma and fibrosis, or xanthogranulomatosis that is CD68 positive and CD1a negative in an immunohistochemical assay [2]. In the case described in this study, the diagnostic resources previously described were used, in addition to MRI monitoring of the skull because of the involvement of the pituitary stalk and the monitoring of disease progression by PET-CT, as suggested in the literature [1]. The treatment proposed for ECD involved the use of interferon- $\alpha$ because among the previously used strategies, the use of interferon- $\alpha$ promoted disease stabilization in most cases [1,13]. The involvement of bones occurs in up to $96 \%$ of patients with ECD. However, bone pain occurs in only $50 \%$ of cases and usually initiates in the region of the knees and ankles [1]. In this case report, bone pain had an insidious start, increased progressively, and was one of the symptoms reported by the patient from the initial evaluation. The pathophysiology of bone pain in ECD involves bilateral and symmetrical osteosclerotic lesions of the diametaphyseal region of long bones. Occasionally, a bone lesion may present as lytic or mixed.

Typically, ECD does not compromise the axial skeleton and the epiphyseal region of the bones [1]. Bone destruction promotes the activation of nociceptors in the periosteum and bone marrow, thus triggering pain [15]. The activation of nociceptors is a result of the local release of inflammatory mediators and reduced $\mathrm{pH}[16,17]$. The treatment used was based on the pathophysiology of bone pain and was guided by the analgesic ladder proposed by the World Health Organization [14]. A treatment regimen comprising a strong opioid associated with an anti-inflammatory and a non-opioid analgesic was initiated. In addition to the specific treatment for bone pain, medications targeting depression and for symptom management were prescribed. For a better assessment of the case, monitoring by a multidisciplinary team was proposed and implemented. After expert evaluation, venlafaxine was incorporated into the treatment of depression. This drug is widely used in the treatment of neuropathic pain and may play a role in the treatment of bone pain [18]. In this respect, a recent study indicated the involvement of neural injury in the pathophysiology of bone pain $[19,20]$. Although pain is an important part of the clinical symptoms of ECD, its characterization and treatment remain understudied, and among the existing studies of ECD, its diagnosis and treatment have been the most studied aspects of the disease. This report has the typical limitations of case reports, with the description of a single patient and his response to the proposed treatment for pain, in addition to the management of symptoms and adverse effects. Because of the reduced number of cases, research on ECD has become severely limited. The poor characterization of pain in ECD most likely stems from the rarity of the syndrome. However, pain has an important role in limiting the quality of life, and its characterization and treatment justify the need for reporting new cases and highlight the importance of sharing the results of different treatments used for pain control. Logically, these data should not be extrapolated to other clinical conditions. To the best of our knowledge, this is the first case report on the characterization and treatment of pain specific to ECD, and we highlight that the patient had a good response to treatment.

\section{References}

1. MazorRD,Manevich-MazorM,ShoenfeldY.Erdheim-ChesterDisease:a comprehensivereview oftheliterature. OrphanetJRareDis. 2013;8:137. PubMed: https://www.ncbi.nlm.nih.gov/pubmed/24011030

2. Haroche J, Arnaud L, Amoura Z. Erdheim-Chester disease. Curr Opin Rheumatol. 2012; 24: 53-59.

PubMed: https://www.ncbi.nlm.nih.gov/pubmed/22089098

3. Braiteh F, Boxrud C, Esmaeli B, Kurzrock R. Successful treatment of Erdheim-Chester disease, a non-Langerhans-cell histiocytosis, with interferon-a. Blood, 2005; 106: 2992-2994.

PubMed: https://www.ncbi.nlm.nih.gov/pubmed/16020507

4. Dagna L, Girlanda S, Langheim S, Rizzo N, Bozzolo EP, et al. Erdheim-Chester disease: report on a case and new insights on its immunopathogenesis. Rheumatology. 2010; 49: 1203-1206. PubMed: https://www.ncbi.nlm.nih.gov/pubmed/20097905

5. Quran S, Reith J, Bradley J, Rimsza L. Erdheim-Chester disease: case report, PCR-based analysis of clonality, and review of literature. Mod Pathol. 2002; 15: 666-672.

PubMed: https://www.ncbi.nlm.nih.gov/pubmed/12065781

6. Murray D, Marshall M, England E, Mander J, Chakera TM. Erdheimchester disease. Clin Radiol. 2001; 56: 481-484.

PubMed: https://www.ncbi.nlm.nih.gov/pubmed/11428798

7. Veyssier-Belot C, Cacoub P, Caparros-Lefebvre D. Erdheim-Chester disease: Clinical and radiologic characteristics of 59 cases. Medicine (Baltimore). 1996; 75: 157-169.

PubMed: https://www.ncbi.nlm.nih.gov/pubmed/8965684

8. Luft T, Pang KC, Thomas E. Type I interferons enhance the terminal differentiation of dendritic cells. J Immunol. 1998; 161: 1947-1953. PubMed: https://www.ncbi.nlm.nih.gov/pubmed/9712065

9. Luft $T$, Luetjens $P$, Hochrein $H$. IFN-alpha enhances CD40 ligandmediated activation of immature monocyte-derived dendritic cells. Int Immunol. 2002; 14: 367-380.

PubMed: https://www.ncbi.nlm.nih.gov/pubmed/11934873

10. Chester W. Über lipoidgranulomatose (Over lipoid granulomatosis). Virchows Arch Pathol Anat Physiol. 1930; 279: 561-602.

11. Haroche J, Arnaud L, Amoura Z. Erdheim-Chester disease. Curr Opin 
Rheumatol. 2012; 24: 53-59.

PubMed: https://www.ncbi.nlm.nih.gov/pubmed/22089098

12. Haroche J, Arnaud L, Cohen-Aubart F, Hervier B, Charlotte F, et al. Erdheim-Chester disease. Rheum Dis Clin North Am. 2013;39: 299-311. PubMed: https://www.ncbi.nlm.nih.gov/pubmed/23597965

13. Kornik RI, Naik HB, Lee CC, Estrada-Veras J, Gahl WA, et al. Diabetes insipidus, bone lesions, and new-onset red-brown papules in a 42-year-old man. J Am Acad Dermatol. 2013; 68: 1034-1038. PubMed: https://www.ncbi.nlm.nih.gov/pubmed/23466246

14. Cavalli G, Guglielmi B, Berti A, Campochiaro C, Sabbadini MG, et al. The multifaceted clinical presentations and manifestations of Erdheim-Chester disease: comprehensive review of the literature and of 10 new cases. Ann Rheum Dis. 2013; 72: 1691-1695.

15. Steinert AF, Reppenhagen S, Baumann B, Rudert M, Nöth U. Total knee arthroplasty in a patient with Erdheim-Chester disease with massive joint destruction: a case report. J Bone Joint Surg Am. 2011; 93: e29. PubMed: https://www.ncbi.nlm.nih.gov/pubmed/21471409
16. Yin JJ, Pollock CB, Kelly K. Mechanisms of cancer metastasis to the bone. Cell Res. 2005; 15: 57-62.

PubMed: https://www.ncbi.nlm.nih.gov/pubmed/15686629

17. Buga S, Sarria JE. The management of pain in metastatic bone disease. Cancer Control. 2012; 19: 154-166.

PubMed: https://www.ncbi.nlm.nih.gov/pubmed/22487978

18. Attal N, Cruccu G, Baron R, Haanpää M, Hansson P, et al. European Federation of Neurological Societies. EFNS guidelines on the pharmacological treatment of neuropathic pain. Eur J Neurol. 2010; 17: 1113-1188.

19. Nascimento D, Pozza DH, Castro-Lopes JM, Neto FL. Neuronal injury marker ATF-3 is induced in primary afferent neurons of monoarthritic rats. Neurosignals. 2011; 19: 210-221.

PubMed: https://www.ncbi.nlm.nih.gov/pubmed/21912089

20. Ivanavicius SP, Ball AD, Heapy CG, Westwood FR, Murray F, et al. Structural pathology in a rodent model of osteoarthritis is associated with neuropathic pain: increased expression of ATF-3 and pharmacological characterisation. Pain. 2007; 128: 272-282. 